\title{
Subclinical myopathic changes in COVID-19
}

\author{
Davide Villa ${ }^{1,2}$. Gianluca Ardolino ${ }^{1}$ - Linda Borellini ${ }^{1}$ (D) . Filippo Cogiamanian ${ }^{1} \cdot$ Maurizio Vergari $^{1}$. \\ Valeria Savojardo $^{3}$. Flora Peyvandi ${ }^{2,4}$. Sergio Barbieri ${ }^{1}$
}

Received: 25 January 2021 / Accepted: 7 July 2021 / Published online: 25 July 2021

(c) Fondazione Società Italiana di Neurologia 2021

\begin{abstract}
Introduction Coronavirus disease 2019 (COVID-19) is associated to neuromuscular symptoms in up to $10.7 \%$ of hospitalized patients. Nevertheless, the extent of muscular involvement in infected subjects with no signs of myopathy has never been assessed with neurophysiological investigations.

Methods Over a 3-week period - from April 30 through May 20, 2020 - a total of 70 patients were hospitalized in the Internal Medicine Ward of the Fondazione IRCCS Ca' Granda Ospedale Maggiore Policlinico in Milan, Italy. After excluding patients who underwent invasive ventilation and steroid treatment, 12 patients were evaluated. Nerve conduction studies (NCS) included the analysis of conduction velocity, amplitude, and latency for bilateral motor tibial, ulnar nerves, and sensory sural and radial nerves. Unilateral concentric-needle electromyography (EMG) was performed evaluating at least 4 areas of 8 selected muscles. For each muscle, spontaneous activity at rest, morphology, and recruitment of motor unit action potentials (MUAPs) were evaluated.

Results While nerve conduction studies were unremarkable, needle electromyography showed myopathic changes in 6 out of 12 subjects. All patients were asymptomatic for muscular involvement. Clinical features and laboratory findings did not show relevant differences between patients with and without myopathic changes.

Conclusion Our data show that in SARS-CoV-2 infection muscular involvement can occur despite the absence of clinical signs or symptoms and should be considered part of the disease spectrum. The application of muscle biopsy to unravel the mechanisms of myofiber damage on tissue specimens could help to clarify the pathogenesis and the treatment response of coronavirus-mediated injury.
\end{abstract}

Keywords COVID-19 $\cdot$ Myopathy $\cdot$ Electromyography $\cdot$ Muscle injury $\cdot$ SARS-Cov-2

Linda Borellini

linda.borellini@gmail.com; linda.borellini@policlinico.mi.it

Davide Villa

davide.villa@unimi.it

Gianluca Ardolino

gianluca.ardolino@policlinico.mi.it

Filippo Cogiamanian

filippo.cogiamanian@policlinico.mi.it

Maurizio Vergari

maurizio.vergari@policlinico.mi.it

Valeria Savojardo

valeria.savojardo@policlinico.mi.it

Flora Peyvandi

flora.peyvandi@policlinico.mi.it
Sergio Barbieri

sergio.barbieri@policlinico.mi.it

1 U.O. Neurofisiopatologia, Fondazione IRCCS Ca' Granda Ospedale Maggiore Policlinico, Via Francesco Sforza 35, Milan, Italy

2 Dipartimento Di Fisiopatologia Medico-Chirurgica E Dei Trapianti, Università Degli Studi Di Milano, Milan, Italy

3 U.O. Medicina Generale Ad Alta Intensità Di Cura, Fondazione IRCCS Ca' Granda Ospedale Maggiore Policlinico, Via Francesco Sforza 35, Milan, Italy

4 Centro Emofilia E Trombosi Angelo Bianchi Bonomi, Fondazione IRCCS Ca' Granda Ospedale Maggiore Policlinico, Via Francesco Sforza 35, Milan, Italy 


\section{Introduction}

Severe acute respiratory syndrome coronavirus 2 (SARSCoV-2) was detected in Wuhan, China, in December 2019. The disease (COVID-19) spread rapidly into a worldwide pandemic causing severe morbidity and mortality. It is mainly considered a respiratory disease, and valuable descriptions about its pulmonary involvement are largely provided by literature. Apart from prominent lung injury, neuromuscular symptoms including muscle weakness and myalgia are encountered in up to $10.7 \%$ of hospitalized patients affected by COVID-19 [1].

Other similar human coronaviruses, such as the Middle East respiratory syndrome virus (MERS-CoV) and the severe acute respiratory syndrome coronavirus 1 (SARSCoV-1), have been previously associated with neurological complications and skeletal muscle injury $[2,3]$. It is tempting to speculate that SARS-Cov-2 may share the same tropism and could lead to neuromuscular damage as its akin viruses.

Cabañes-Martinez and Villadóniga [4] extensively assessed the prevalence and the characteristics of neuromuscular involvement in twelve intensive care unit (ICU) patients affected by COVID-19. Signs of critical illness myopathy (CIM) and polyneuropathy (CIP) were documented in 11 out of 12 patients with nerve conduction studies (NCS), quantitative EMG, and muscle biopsy, although no distinctive pattern of neuromuscular involvement was found. In line with these results, Versace and Bagnato described 3 cases of CIM after SARS-Cov-2 infection and came to similar conclusions $[5,6]$.

CIM has been described as a potential confounding factor during the identification of SARS-Cov-1-related myopathy [3]; in addition to that, the ICU setting and the need of personal protection equipment may compromise the accurate detection of myopathic involvement in such patients.

To precisely assess the extent of primary neuromuscular involvement in SARS-Cov-2 infection, we focused on a subset of non-severe COVID-19 patients.

\section{Methods}

The study was approved by the ethics committee of Fondazione IRCCS Ca' Granda Ospedale Maggiore Policlinico, and written informed consent was obtained from all patients.

Over a 3-week period - from April 30 through May 20, 2020 - a total of 70 patients affected by COVID-19 were hospitalized in one of the Internal Medicine wards of the Fondazione IRCCS Ca' Granda Ospedale Maggiore Policlinico in Milan, Italy.
We focused on patients aged from 18 to 80 years. COVID19 diagnosis was confirmed with polymerase chain reaction on at least 2 nasopharyngeal swabs performed before and after our evaluation.

To avoid the inclusion of patients affected by intensive care unit acquired weakness (ICUAW) that can be caused either by critical illness myopathy (CIM) or polyneuropathy (CIP) [7], we excluded patients who underwent invasive ventilation. Patients undergoing steroid treatment were also excluded to avoid misinterpretation of data due to the possibility of steroid-induced myopathy.

Twelve patients fulfilling these criteria were enrolled.

We tested muscle strength in functional limb muscle groups with the Medical Research Council (MRC) scale; then, we combined individual MRC scores into a sum score, in order to yield an overall estimation of motor function. This score was defined as the sum of MRC from six muscle groups in the upper and lower limbs on both sides so that the score ranged from 60 (normal) to 0 (quadriplegic). Each of the six muscle groups (shoulder abductors, elbow flexors, wrist extensor, hip flexor, knee extensors, foot dorsiflexors) was examined bilaterally each with a score from 0 to 5 [8]. Neurological evaluation and MRC sum score were performed by a trained neurologist immediately before the neurophysiological investigation that was carried out by 2 different trained neurophysiologists in a double-blind assessment.

The patients' medical records were reviewed with particular attention to their clinical presentations, laboratory findings, COVID-19-related therapies, and clinical courses.

Nerve conduction studies (NCS) were performed in all patients; the protocol included the analysis of conduction velocity, amplitude and latency for bilateral motor tibial, ulnar nerves and sensory sural and radial nerves. Sensory NCS were conducted with antidromic stimulation.

Concentric-needle electromyography (EMG) was performed evaluating at least 4 areas of each muscle tested. For all subjects, the electromyographic protocol included unilateral examination of the following muscles: trapezius, deltoid, extensor digitorum communis, first dorsal interosseus, iliopsoas, vastus medialis, tibialis anterior, and gastrocnemius medialis. For each muscle, spontaneous activity at rest, morphology, and recruitment of motor unit action potentials (MUAPs) were evaluated.

Quantitative EMG was performed when possible: mean amplitude and duration of motor unit potentials (MUP) were measured, as well as the percentage of polyphasic potentials. The recruitment pattern was also evaluated in those cases where the clinical situation of the patient allowed it. EMG interpretation was completed by 2 trained neurophysiologists immediately after the conclusion of the respective measurement, based on the characteristics of the EMG waveforms (high-pass filter set to $10 \mathrm{~Hz}$, low-pass filter setting $10 \mathrm{kHz}$ ). 
Epochs of $200 \mathrm{~ms}$ were displayed on-screen by setting the sweep speed to $10 \mathrm{~ms} /$ division.

\section{Results}

A total of 12 patients were included in this study. Mean age was 69 (SD 12.80) years, and 7 patients were women. None of the patients exhibited neuromuscular symptoms during the disease course. Reported symptoms were as follows: fever $(10(83 \%))$, cough $(8(67 \%))$, dyspnea $(6(50 \%))$, diarrhea $(4(33 \%))$, and conjunctivitis $(2(17 \%))$. All patients showed mild to moderate respiratory symptoms and chest $\mathrm{X}$ rays were suggestive of pneumonia. The main comorbidity was hypertension (6 (50\%)). Overall, 3 patients (25\%) were started on hydroxychloroquine at admission, 3 (25\%) were started on remdesivir and only one (8\%) was treated with both. Four patients (33\%) received statins before hospitalization.

Neurological examination was unremarkable and all the subjects obtained a MRC sum score of 60 .

None of the patients showed an elevation of the serum creatine phosphokinase (CPK) (mean 59.9-SD 37.18) or serum lactate dehydrogenase (LDH) (mean 298.3-SD 165.20) levels performed before the neurophysiological assessment. Compared to laboratory normative values, our population had high mean levels of C-reactive protein (CRP) (mean 2.54 mg/dL-SD 2.67), D-dimer (mean $2570 \mu \mathrm{g} / \mathrm{L}-\mathrm{SD}$ 1778.88), fibrinogen (mean $535 \mu \mathrm{g} / \mathrm{L}-\mathrm{SD} 131.93$ ), and ferritin (mean $900 \mu \mathrm{g} / \mathrm{dL}-\mathrm{SD} 488.67$ ).

Clinical evaluation and neurophysiological assessment were performed from 5 to 48 (mean 21.83-SD 15.25) days after symptoms onset.

The clinical features and the laboratory findings of the cohort are summarized in Table 1.

No NCS abnormalities were found except the absence of bilateral sural sensory action potential (SAP) in patient 12 , which was already reported in a previous evaluation and likely due to diabetes.

Short-duration, low-amplitude, polyphasic, and highpitch MUAPS with a characteristic early recruitment pattern as described by Dauber and Rubin [9] were documented in 6 out of 12 subjects. All of these studies were concluded as having electromyographic signs of myopathy. Such alterations were prominent in a patchy fashion, in particular in proximal muscles such as trapezius (6 (50\%)), iliopsoas (4 $(33 \%)$ ), and deltoid (1 (8\%)). No spontaneous activities were recorded (Fig. 1 and Supplementary Material).

Given the exiguity of our cohort, we could not perform statistical correlations between muscle involvement, demographic data, pharmacological treatment, and blood tests. However, the levels of fibrinogen, CPK, D-dimer, and C-reactive protein did not show relevant differences between the two groups. When compared to non-myopathic subjects, patients with myopathic alterations exhibited more severe respiratory conditions according to the need for non-invasive mechanic ventilation (NIMV).

\section{Discussion}

The present report documents subclinical myopathic changes in 6 out of 12 patients infected with SARS-Cov-2. To our knowledge, this is the first description of muscular involvement with neurophysiological assessment in COVID-19-affected patients with no signs of myopathy.

Other coronavirus infections have been previously associated with skeletal muscle injury, despite viral cultures yielding no organisms [3]. A metallopeptidase named angiotensin-converting enzyme 2 (ACE2) has been identified as the functional receptor for SARS-Cov-1 and SARS-Cov-2. SARS-Cov-2 spike protein directly binds with the host cell surface ACE2 receptor promoting virus entry and replication [10]. ACE2 expression in skeletal muscle has been previously reported [11] suggesting that SARS-Cov-2 may have skeletal muscle tropism directly causing muscular damage. Another possible mechanism of muscular involvement could imply an overactive immune state promoted by cytokines storm and molecular mimicry between specific viral and muscular proteins [12]. Inflammatory cytokine storm in addition with coagulopathy and macrophage activation has been reported as the primary pathogenetic mechanism that causes interstitial pneumonia and damage to extrapulmonary tissues and organs in COVID-19; skeletal muscular damage may be due to the same process [5, 13]. Cytokines play a key role in initiating, propagating, and regulating tissuespecific autoimmune injury. This pathway is thought to be implied in two-thirds of Guillain Barré syndrome (GBS) cases [14]. Five GBS cases have been documented in Italy after SARS-Cov-2 infection $[15,16]$ although a clear causeeffect relation has not been elucidated yet [17]. Furthermore, both acute motor axonal neuropathy (AMAN) and acute inflammatory demyelinating polyneuropathy (AIDP) variants have been reported after SARS-Cov-1 infections [18]. However, in our population, myopathic patients do not seem to exhibit higher inflammatory state compared to nonmyopathic subjects.

In a recent case series, Mao and colleagues described muscular involvement in $19.3 \%$ of severe and $4.8 \%$ of nonsevere COVID-19 patients [1]. These authors defined as "skeletal muscle injury" the presence of both muscle pain and elevated CPK serum level (>200 U/L). However, in our cohort, despite myopathic changes on EMG, the neurological examination including MRC sum score was normal in all subjects, as was the CPK serum level performed before the neurophysiological assessment. Furthermore, motor 


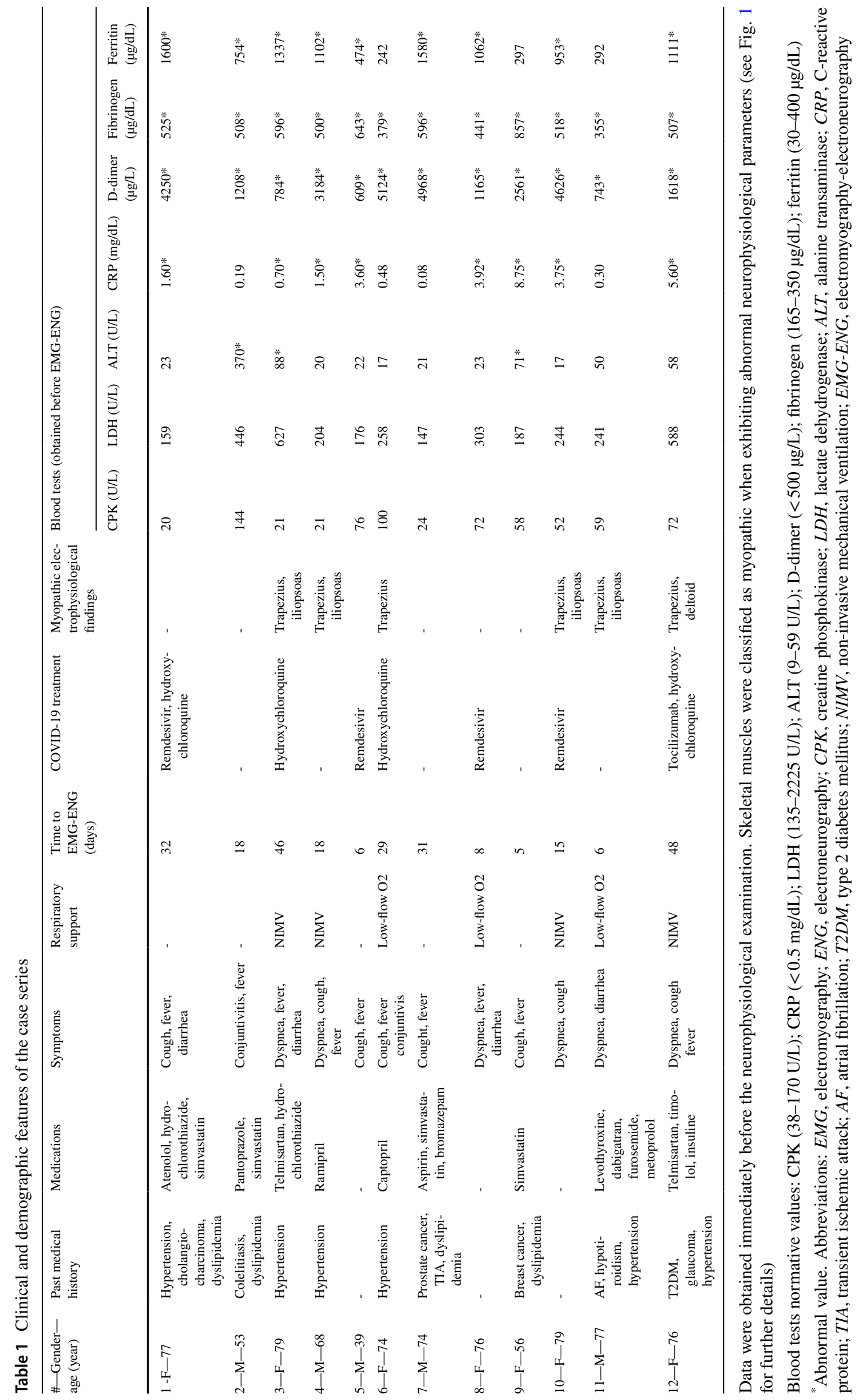


Fig. 1 Myopathic pattern: short duration, small amplitude (mean: $3.9 \mathrm{~ms}, 319 \mu \mathrm{V}$ ) and polyphasic motor unit potentials of the trapezius muscle in patient 11

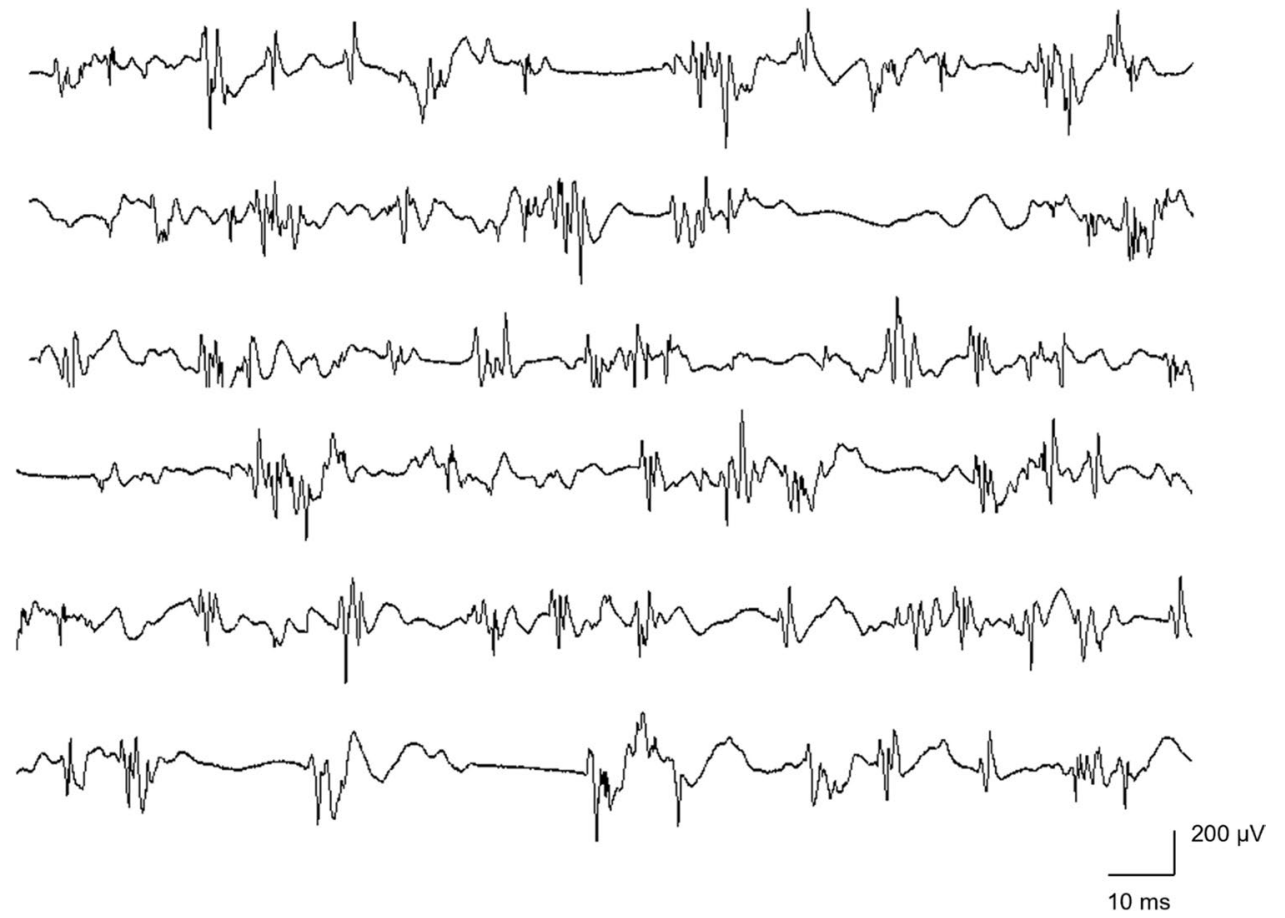

peripheral neuropathy with bilateral low limb weakness has been well described by Abdelnour et al. as a presenting manifestation in SARS-Cov-2 infection [19]. When comparing our results to those of Mao and Abdelnour, it must be pointed out that they did not perform EMG to quantify skeletal muscle injury. Despite the good accuracy [8], the MRC grading system and related multi-item constructed sum-scores have a methodological shortcoming that could lead to examiners' disagreement mainly regarding scores 4 and 5 [20]. A possible explanation could be that during MRC administration the resistance applied by the examiner to differentiate between scores 4 and 5 is somehow arbitrary. The need of personal protection equipment may have interfered with clinical evaluation; therefore, we cannot exclude to have incurred in misclassification between scores 4 and 5 . Since it is challenging to identify subtle muscular weakness with MRC grading system, in our cohort, EMG may have been helpful in unravelling MUAP alterations in affected muscles that are not weak on neurologic examination [21].

CPK levels are typically increased in muscle necrosis or muscle membrane leakage, although direct correspondence between the laboratory and EMG findings is not consistently found [22]. In our population, myopathic pattern was documented only in limited areas of each affected muscle, suggesting a patchy myofiber involvement. It is likely that CPK serum level sensitivity is lower in such a scenario. In line with our results, Daia et al. have hypothesized a temporary myopathic injury without persistently elevated enzymes after SARS-Cov-2 infection [23].
To date, COVID-19 treatment is not standardized; thus, our patients underwent different drug protocols. We cannot exclude a priori the role of pharmacological treatments as a possible confounder in our findings. Hydroxychloroquine may interfere with viral entry via ACE 2 binding [24, 25]. Irritability, psychosis, peripheral neuropathy, and myopathy are reported as possible neurologic adverse effects [26, 27]. In our cohort, 4 patients were started on hydroxychloroquine; however, none of them exhibited myopathic changes at neurophysiological assessment. Remdesivir, a nucleotide analogue prodrug that inhibits viral RNA polymerases, has shown in vitro activity against SARS-Cov-2 [28]. Literature about potential neuromuscular adverse effects is scant. In patients with severe inflammatory disease, cytokine release may be reduced by tocilizumab administration [29]. To our knowledge, no muscular adverse effects are reported. Statin treatment can make skeletal muscles susceptible to myopathy and consequently alter electromyographic results [30]. However, simvastatin was taken by 4 patients in our cohort and none of them showed myopathic pattern on EMG.

The main limitation of this study is the small size of our cohort that prevented us to assert actual prevalence of neuromuscular involvement in patients affected by COVID-19. In order to achieve the best reliability of our analyses, we settled rigid inclusion criteria to avoid misclassification, above all ICUAW and steroid myopathy. Even though limiting the power our analysis, the possibility to focus on a homogeneous subgroup allowed us to minimize the effect of all possible confounders. 
In addition to that, the latency between COVID-19 symptoms onset and neurophysiological evaluation was heterogeneous among all subjects. In fact, this limitation forbade us to unravel the actual onset as well as the evolution of COVID-19-related muscular injury. The employment of EMG in a wider spectrum of patients including different disease severity and the longitudinal analysis of myopathic changes could shed light on the natural history of this condition. Fatigue and musculoskeletal injury are reported in both ICU and non-ICU patients 4 weeks after discharge from hospital as described by Halpin in the post-COVID syndrome [31]. However, predictive factors for developing post-COVID syndrome are not well established yet. It is tempting to speculate that myopathic damage could play a key role in developing musculoskeletal symptoms later, such as weakness, pain, and fatigue. In this scenario, EMG may help to early identify rehabilitation needs and maximize the functional return of patients infected by SARS-Cov-2. Nevertheless, the analysis of tissue specimen from muscle biopsy seems to be necessary to ascertain the actual mechanisms of myofiber damage.

Another limitation of this study is the lack of a control group represented by patients admitted to a Medicine Ward for pathologies different from COVID-19. Therefore, the abnormalities reported may be due to the simple hospitalization, drugs, or concurrent pathologies. However, to our best knowledge, no data regarding patients without muscular injury admitted to a medicine ward developing myopathic pattern on EMG are actually available in literature.

In conclusion, our data show that muscular involvement occurs in SARS-Cov-2 infection despite the absence of clinical signs or symptoms and should be considered part of the disease spectrum. Further studies are warranted to document the actual prevalence of this manifestation, as to clarify the pathogenesis and the treatment response of COVID-19 myopathy.

Supplementary Information The online version contains supplementary material available at https://doi.org/10.1007/s10072-021-05469-8.

Author contribution All authors have contributed to the work, as well as read and approved the final manuscript.

\section{Declarations}

Ethical approval All authors have read the Journal's Position on Issues involved in Ethical Publication and affirm that this report is consistent with those guidelines.

Conflict of interest The authors declare no competing interests.

\section{References}

1. Mao L, Jin H, Wang M, Hu Y, Chen S, He Q, Chang J, Hong C, Zhou Y, Wang D, Miao X, Li Y, Hu B (2020) Neurologic manifestations of hospitalized patients with coronavirus disease 2019 in Wuhan, China. JAMA Neurol 77(6):683-690. https:// doi.org/10.1001/jamaneurol.2020.1127

2. Arabi YM, Harthi A, Hussein J et al (2015) Severe neurologic syndrome associated with Middle East respiratory syndrome corona virus (MERS-CoV). Infection 43(4):495-501. https:// doi.org/10.1007/s15010-015-0720-y

3. Leung TW, Wong KS, Hui AC et al (2005) Myopathic changes associated with severe acute respiratory syndrome: a postmortem case series. Arch Neurol 62(7):1113-1117

4. Cabañes-Martínez L, Villadóniga M, González-Rodríguez L, Araque L, Díaz-Cid A, Ruz-Caracuel I, Pian H, Sánchez-Alonso S, Fanjul S, Del Álamo M, Regidor I (2020) Neuromuscular involvement in COVID-19 critically ill patients. Clin Neurophysiol 131(12):2809-2816. https://doi.org/10.1016/j.clinph. 2020.09.017

5. Versace V, Sebastianelli L, Ferrazzoli D, Saltuari L, Kofler M, Löscher W, Uncini A (2021) Case report: Myopathy in critically ill COVID-19 patients: A Consequence of Hyperinflammation? Front Neurol 29(12):625144. https://doi.org/10.3389/ fneur.2021.625144

6. Bagnato S, Boccagni C, Marino G, Prestandrea C, D'Agostino T, Rubino F (2020) Critical illness myopathy after COVID-19. Int J Infect Dis 99:276-278. https://doi.org/10.1016/j.ijid.2020.07.072

7. Hermans G, Van den Berghe G (2015) Clinical review: intensive care unit acquired weakness. Crit Care. 19(1):274. https://doi. org/10.1186/s13054-015-0993-7

8. Dyck PJ, Boes CJ, Mulder D, Millikan C, Windebank AJ, Dyck PJ et al (2005) History of standard scoring, notation, and summation of neuromuscular signs. A current survey and recommendation. J Peripher Nerv Syst 10:158-173

9. Rubin J (2009) Daube, JR, Devon I. Needle electromyography. Muscle Nerve 39:244-270. https://doi.org/10.1002/mus.21180

10. Zhang H, Penninger JM, Li Y et al (2020) Angiotensin-converting enzyme 2 (ACE2) as a SARS-CoV-2 receptor: molecular mechanisms and potential therapeutic target. Intensive Care Med 46:586-590. https://doi.org/10.1007/s00134-020-05985-96

11. Li MY, Li L, Zhang Y, Wang XS (2020) Expression of the SARS-CoV-2 cell receptor gene ACE2 in a wide variety of human tissues. Infect Dis Poverty 9(1):1-7

12. Ye Q, Wang B, Mao J (2020) The pathogenesis and treatment of the 'Cytokine storm' in COVID-19. J Infect 80(6):607613. https://doi.org/10.1016/j.jinf.2020.03.037

13. McGonagle D, Sharif K, O'Regan A, Bridgewood C (2020) The role of cytokines including Interleukin-6 in COVID-19 induced pneumonia and macrophage activation syndrome-like disease. Autoimmun Rev 19(6):102537. https://doi.org/10.1016/j.autrev. 2020.102537

14. Lu M, Zhu J (2011) The role of cytokines in Guillain-Barré syndrome. J Neurol 258:533-548. https://doi.org/10.1007/ s00415-010-5836-5

15. Toscano G, Palmerini F, Ravaglia S et al (2020) Guillain-Barré syndrome associated with SARS-CoV-2. NEnglJMed. https:// doi.org/10.1056/NEJMc2009191

16. Agosti E, Giorgianni A, D'Amore F et al (2021) Is GuillainBarrè syndrome triggered by SARS-CoV-2? Case report and literature review. Neurol Sci 42:607-612. https://doi.org/10. 1007/s10072-020-04553-9

17. Finsterer J, Scorza CA, Scorza FA, Fiorini AC (2020) Is Guillain Barre syndrome truly caused by SARS-CoV-2? Am J Emerg 
Med S0735-6757(20):30983-30989. https://doi.org/10.1016/j. ajem.2020.11.002

18. Tsai LK, Hsieh ST, Chao CC et al (2004) Neuromuscular disorders in severe acute respiratory syndrome. Arch Neurol 61(11):16691673. https://doi.org/10.1001/archneur.61.11.1669

19. Abdelnour L, EltahirAbdalla M, Babiker S (2020) COVID 19 infection presenting as motor peripheral neuropathy. J Formos Med Assoc 119(6):1119-1120. https://doi.org/10.1016/j.jfma. 2020.04.024

20. Hermans G, Clerckx B, Vanhullebusch T, Segers J, Vanpee G, Robbeets $C$ et al (2012) Interobserver agreement of medical research council sum-score and handgrip strength in the intensive care unit. Muscle Nerve 45:18-25

21. Paganoni S, Amato A (2013) Electrodiagnostic evaluation of myopathies. Phys Med Rehabil Clin N Am 24(1):193-207. https://doi. org/10.1016/j.pmr.2012.08.017

22. David Lacomis MD (2012) Electrodiagnostic approach to the patient with suspected myopathy. Neurol Clin 30:641-660. https:// doi.org/10.1016/j.ncl.2011.12.007

23. Daia C, Scheau C, Neagu G, Andone I, Spanu A, Popescu C, Stoica SI, Verenca MC, Onose G (2021) Nerve conduction study and electromyography findings in patients recovering from Covid19 - case report. Int J Infect Dis 103:420-422. https://doi.org/10. 1016/j.ijid.2020.11.146

24. Vincent MJ, Bergeron E, Benjannet $\mathrm{S}$ et al (2005) Chloroquine is a potent inhibitor of SARS coronavirus infection and spread. Virol J 2:69. https://doi.org/10.1186/1743-422X-2-69

25. Zhou D, Dai SM, Tong Q (2020) COVID-19: a recommendation to examine the effect of hydroxychloroquine in preventing infection and progression. J Antimicrob Chemother 75(7):1667-1670. https://doi.org/10.1093/jac/dkaa114

26. Tönnesmann E, Kandolf R, Lewalter T (2013) Chloroquine cardiomyopathy - a review of the literature. Immunopharmacol Immunotoxicol 35(3):434-442. https://doi.org/10.3109/08923 973.2013.780078
27. Manzo C, Gareri P, Castagna A (2017) Psychomotor agitation following treatment with hydroxychloroquine. Drug Saf - Case Rep 4:6. https://doi.org/10.1007/s40800-017-0048-x

28. Grein J, Ohmagari N, Shin D, Diaz G, Asperges E, Castagna A, Feldt T, Green G, Green ML, Lescure FX, Nicastri E, Oda R, Yo K, Quiros-Roldan E, Studemeister A, Redinski J, Ahmed S, Bernett J, Chelliah D, Chen D, Chihara S, Cohen SH, Cunningham J, D’Arminio Monforte A, Ismail S, Kato H, Lapadula G, L'Her E, Maeno T, Majumder S, Massari M, Mora-Rillo M, Mutoh Y, Nguyen D, Verweij E, Zoufaly A, Osinusi AO, DeZure A, Zhao Y, Zhong L, Chokkalingam A, Elboudwarej E, Telep L, Timbs L, Henne I, Sellers S, Cao H, Tan SK, Winterbourne L, Desai P, Mera R, Gaggar A, Myers RP, Brainard DM, Childs R, Flanigan $\mathrm{T}$ (2020) Compassionate use of remdesivir for patients with severe Covid-19. N Engl J Med 382(24):2327-2336. https://doi.org/10. 1056/NEJMoa2007016

29. Zubair AS, McAlpine LS, Gardin T, Farhadian S, Kuruvilla DE, Spudich S (2020) Neuropathogenesis and neurologic manifestations of the coronaviruses in the age of coronavirus disease 2019: a review. JAMA Neurol 77(8):1018-1027. https://doi.org/10.1001/ jamaneurol.2020.2065

30. Lotteau S, Ivarsson N, Yang Z et al (2019) A mechanism for statin-induced susceptibility to myopathy. JACC Basic Transl Sci 4(4):509-523. https://doi.org/10.1016/j.jacbts.2019.03.0125

31. Halpin SJ, McIvor C, Whyatt G, Adams A, Harvey O, McLean L, Walshaw C, Kemp S, Corrado J, Singh R, Collins T, O'Connor RJ, Sivan M (2020) Postdischarge symptoms and rehabilitation needs in survivors of COVID-19 infection: A cross-sectional evaluation. J Med Virol 93(2):1013-1022. https://doi.org/10.1002/jmv.26368

Publisher's note Springer Nature remains neutral with regard to jurisdictional claims in published maps and institutional affiliations. 